\title{
Measurement of the vibration state of an array of micro cantilevers by diffractional methods
}

\section{Medida del estado de vibración de un array de micro-voladizos mediante métodos difraccionales}

\author{
A. Cuadrado(1,*), J. Agustí(2), M. López-Suárez(2), G. Murillo(2), G. Abadal(2), J. Alda(1,S) \\ 1. Applied Optics Complutense Group. University Complutense of Madrid. School of Optics. Av. Arcos de Jalón, 118. \\ 28037 Madrid. Spain. \\ 2. Departament d'Enginyeria Electrònica, Universitat Autònoma de Barcelona (UAB), Bellaterra, 08193 Barcelona, \\ Spain. \\ ("Email: a.cuadrado@pdi.ucm.es $\quad$ S: miembro de SEDOPTICA / SEDOPTICA member \\ Recibido / Received: 16/09/2011. Revisado / Revised: 14/12/2011. Aceptado / Accepted:16/12/2011.
}

\begin{abstract}
:
The amplitude of vibration of an array of micro-cantilevers piezoelectrically excited has been measured by analyzing the diffraction pattern given by the structure. A model for the simulation of this situation, and some out-of-plane interferometric measurements based on diffraction pattern, have been developed and performed to fully understand the dynamic of the elements and the diffraction pattern produced by them.
\end{abstract}

Key words: Diffraction, Micro-Electro-Mechanical Systems.

\section{RESUMEN:}

En esta contribución, se analiza la amplitud de vibración de un array de micro-voladizos excitados a través de un piezoeléctrico. El objeto de análisis son las distribuciones de irradiancia generadas por la estructura. Se ha desarrollado un modelo que explica los cambios producidos en las figuras de y se han realizado medidas de la figura de difracción producida. Esto permite un mejor conocimiento de la dinámica del dispositivo y la generación de la figura de difracción.

Palabras clave: Diffraction, Micro-Electro-Mechanical Systems.

\section{REFERENCIAS Y ENLACES / REFERENCES AND LINKS}

[1]. G. M. Rebeitz, RF MEMS: Theory, Design and Technology, John Wiley \& Sons, Inc (2003).

[2]. K. L. Ekinci, Y. T. Yang, M. L. Roukes, "Ultimate limits to inertial mass sensing based upon nanoelectromechanical systems", J. Appl. Phys. 95, 2682-2689 (2004)

[3]. Y. Qin, X. Wang, Z. L. Wang, "Microfibre-nanowire hybrid structure for energy scavenging", Nature 451, 809-814 (2008)

[4]. K. Jensen, J. Weldon, H. Garcia, A. Zettl, "Nanotube radio", Nano Lett. 8, 374-374 (2008).

[5]. M. E. Motamedi, MOEMS - Micro-Opto-Electro-Mechanical Systems, SPIE Press(2005).

[6]. D. Okawa, S. J. Pastine, A. Zettl, J. M. J. Frechet, "Surface tension mediated conversion of light to work", J. Am. Chem. Soc. 131, 5396-5398 (2009).

[7]. J. Alda, J. M. Rico-García, J. M. López-Alonso, G. Boreman, "Optical antennas for nanophotonic applications", Nanotechnology 16, S230-S234 (2005).

[8]. D. Karabacak, T. Kouch, K. L. Ekinci, "Analysis of optical interferometric displacement detection in nanoelectromechanical systems", J. Appl. Phys. 98, 124309 (2005).

[9]. D. Karabacak, T. Kouch, C. C. Huang, K. L. Ekinci, "Optical knife-edge technique for nanomechanical displacement detection", Appl. Phys. Lett. 88, 193122 (2006). 
[10].J. Casas, Óptica, Librería Pons (1983).

[11]. J. W. Goodman, Introduction to Fourier Optics, $3^{\text {rd }}$ edition, Roberts \& Company (2004).

[12]. E. Hecht, Optics, $4^{\text {th }}$ edition, Addison Wesley (2001).

\section{Introduction}

The use of micro-electromechanical systems (MEMS) and their nano-scaled relatives (NEMS) [1] have opened the way for innovative applications in several areas, including medicine, communications, or aerospatial applications. Most recently, they have appear in the energy conversion arena [2-5]. Although the use of MEMS in optics is already known [6], the advanced integration of photonic functionalization of MEMS and NEMS should expand their range of possible stimuli to the optical field. This link between photonics and electromechanics at the nanometric scale is addressed by the authors in the form of optical antennas coupled to MEMS and NEMS [7]. The interaction of light may also serve as a characterization tool of the mechanical properties of the electromechanical devices. This is the topic covered in this paper where optics enables the accurate measurement of vibrating MEMS structures.

The movement of MEMS can be optically interrogated by illuminating them and registering their diffraction pattern $[8,9]$. This contribution analyzes this problem, both theoretically and experimentally. We propose a diffractional method able to identify the amplitude and vibration mode of a set of cantilevers. The result of the method has been compared with experimental data obtained from actual devices working within a range of voltages. The images of the diffractive patterns have been properly analyzed to extract the desired parameters. At the same time we may identify the location of a single fast detector able to follow in real time the vibration of a single cantilever. The results obtained here may serve to better understand the effect of the coupling between adjacent cantilevers vibrating in a variety of modes.

In section 2 of this paper we briefly describe the cantilevers device, showing their size and dimension, and presenting the operational mode along with the equation describing the expected profile. Section 3 explains the experimental set- up used in these measurements. In section 4 we adapt the diffraction model to the case of the cantilever measurement. In this treatment we calculate the diffraction produced by a curved surface having a profile given by the vibrating modes. Then, in section 5, the experimental results are compared with the analytical predictions. Finally, section 6 summarizes the main results of this contribution.

\section{Dimensional and mechanical characterization of MEMs}

\subsection{Dimensional values of MEMs}

The MEMs devices under test are built as a set of cantilevers. They are one-side clamped small metallic beams. The bars are connected to a piezoelectric transducer located at the junction with the substrate. The system operates by electrically driving the piezoelectric to mechanically excite the cantilevers. Figure 1 shows a microphotography of the structure. In this figure $H$ is the cantilever length, which is equal to $220 \mu \mathrm{m}, d$ is $30 \mu \mathrm{m}, t$ is the width of the cantilever, being $40 \mu \mathrm{m}$; the gap between two bars is labeled as $s$ and it is $120 \mu \mathrm{m}$; and finally, the thickness of the cantilever is $3.55 \mu \mathrm{m}$.

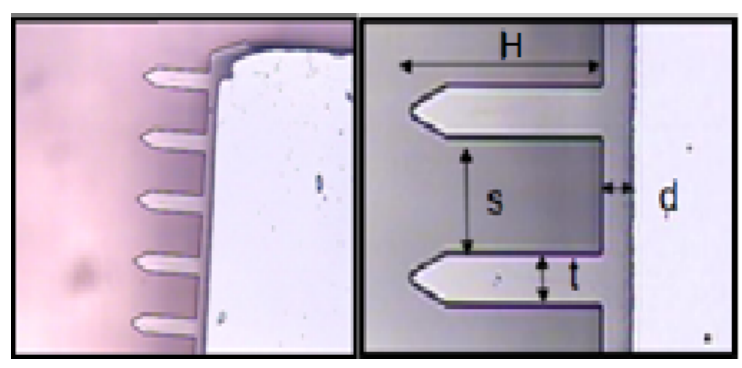

Fig. 1. Microphotography of the cantilever's array analyzed in this paper.

\subsection{Mechanical modes}

The profile of a moving cantilever depends on its vibration mode. The movement modes are those of a free moving one-side-clamped beam [1]. The relations are: 


$$
\begin{array}{r}
z(y)=\sin (k n \times y)-\sinh (k n \times y) \\
-\frac{1}{k k}(\cos (k n \times L) \\
-\cosh (k n \times L)),
\end{array}
$$

where the constant $k k$ is given as,

$$
k k=\frac{\sin (k n \times L)-\sinh (k n \times L)}{\cos (k n \times L)+\cosh (k n \times L)} .
$$

The value of $k n \times L$ depends on the vibration mode, being 1.875 for the first mode, 4.69 for the 2nd, and 7.85 for the third. Figure 2 shows the normalized shape (to the maximum amplitude) of a cantilever vibrating in the first mode as a function of its normalized length. This vibration amplitude can be obtained by assuming a massspring model that is harmonically excited by an external force.

\section{Experimental set up}

The diffractive pattern is obtained and registered using the optical setup described in Fig. 3. A He-Ne laser beam $(\lambda=632.8 \mathrm{~nm})$ illuminates the array of cantilevers with an incident angle ranging from $25^{\circ}$ to $45^{\circ}$. The array of cantilevers is mounted on a xyz translation stage, and the generated diffractive pattern is projected onto a flat screen placed at a distance ranging from $0.5 \mathrm{~m}$ to $1 \mathrm{~m}$ (see Fig. 3(a)). As it is shown in the inset of Fig. 3(a), the diffractive pattern can be easily resolved because, when placing the screen at $1 \mathrm{~m}$, the period of the diffractive pattern is around $4 \mathrm{~mm}$. Alternatively, the diffraction pattern can be captured using a CCD camera placed at shorter distance from the cantilevers than the projection screen (see Fig. 3(b)). In this case the image detected by the camera (Fig. 3(c)) has the same characteristics than the one obtained from the screen projection method (Figure 3(a)).

\section{Diffraction theory}

In this section we describe the diffraction calculation needed to simulate the irradiance distribution at the screen or on the camera locations [10-12]. This model takes into account the dimensions of the system and the incident laser wavelength of $632.8 \mathrm{~nm}$. It also explains

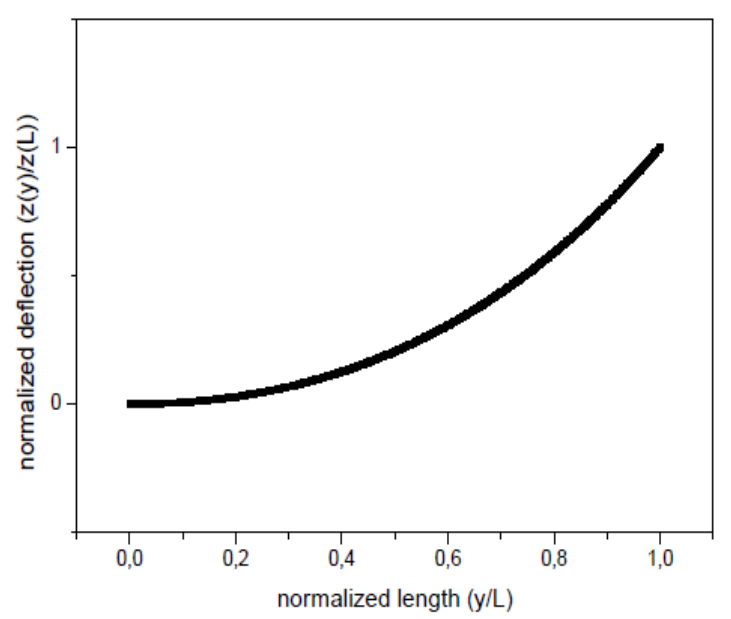

Fig. 2. Profile of the maximum amplitude of a cantilever vibrating in the 1st mode (see Eq. (1)).

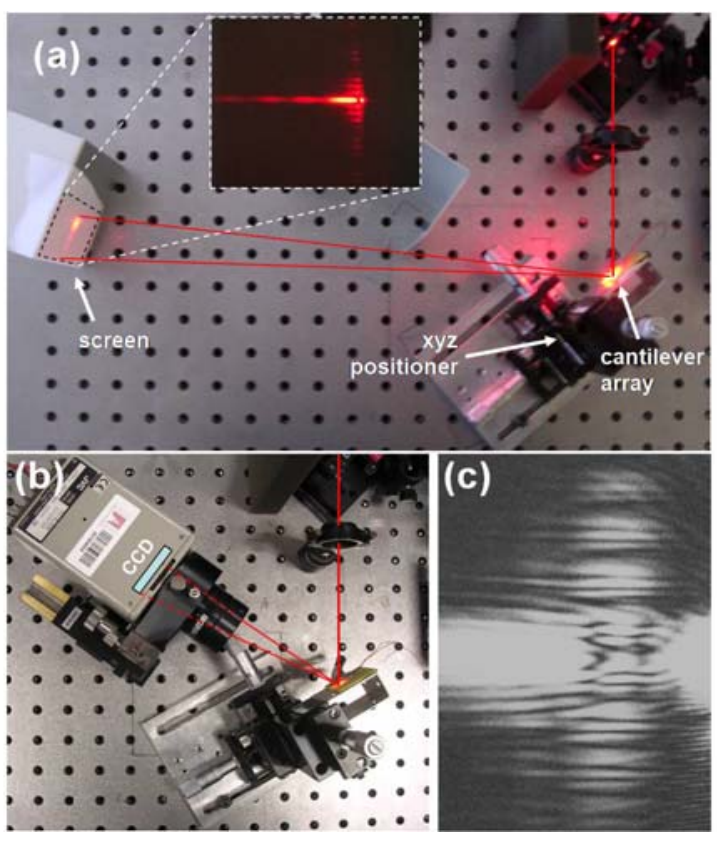

Fig. 3. Experimental set-up for the measurement of the diffraction pattern given by a vibrating cantilever array. The set-up (a) produces an image on a screen. If we want to register the image directly on the CCD we use the set-up (b) that produces images as (c).

the observed diffraction pattern when the cantilevers are in motion.

The MEMS structure under study, when in rest, can be approximated by a set of slits. This is a typical case solved and studied within Fraunhofer diffraction theory. Using this approach, the diffraction intensity pattern produced by $N$ slits is described by the following equation: 


$$
I=I_{0}\left[\frac{\sin \left(\frac{1}{2} H k \sin \theta\right)}{\frac{1}{2} H k \sin \theta}\right]^{2} \cdot\left[\frac{\sin \left(\frac{1}{2} t k \sin \alpha\right)}{\frac{1}{2} t k \sin \alpha}\right]^{2} \cdot\left[\frac{\sin \left(\frac{1}{2} N b k \sin \alpha\right)}{\sin \left(\frac{1}{2} b k \sin \alpha\right)}\right]^{2}
$$

where $H$ is the length of the cantilever, $t$ is its width, $b$ is the period of the cantilevers, and $k=2 \pi / \lambda$, being $\lambda$ the wavelength.

Equation (3) contains two angle variables: $\alpha$ and $\theta$, which describe the angles with respect to the $X$ and $Y$ axis respectively. These angles are shown in Fig. 4.

The first term in Eq. (3) refers to the diffraction produced by a slit with width $H$ along the longitudinal axis direction. The second term has the same form, but it refers to the transversal axis direction, where the slit width coincides with the cantilever width, $t$. Finally the
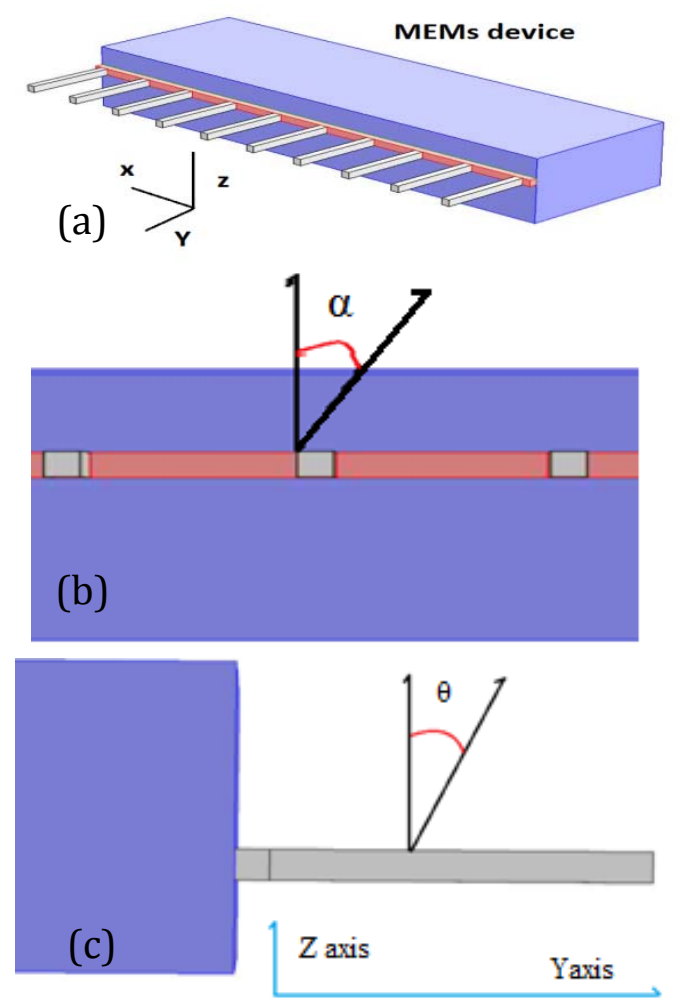

Fig. 4. Graphical layout of the geometrical arrangement of the array of cantilevers. a) 3D view of the MEMS device, b) frontal view where $\alpha$ angle is show, and c) lateral view showing $\theta$. third term is the interference produces by $N$ slits in the array spaced with period $b$.

Figure 2 shows the profile of the first mode. In this situation light will reflect with an angle $\beta$, that is related with the slope of the profile, and with the incident angle. In case of normal incidence, the angle $\beta$ will be:

$$
\beta(y)=2 \tan ^{-1}\left[A \frac{d z(y)}{d y}\right]
$$

where $z(y)$ is the normalized profile function defined in Eq. (1). And $A$ is the maximum amplitude of the cantilever for this profile.

To compute the coherent sum of the different reflected fields, we need the calculation of the phase difference between them. We will consider the position $y=0$ as the location where the bar is clamped. On the other hand, the phase shift, $\delta$, is related with the local height along the bar, $A z(y)$, as

$$
\delta(y)=2 \frac{2 \pi}{\lambda} A z(y),
$$

where the additional 2 factor accounts for the reflection mode that is applied here. After computing the coherent sum we obtain the diffraction pattern generated by a given profile. However, the cantilevers are harmonically moving and therefore the constant $A$ will follow a sinusoidal function with frequency equal to the vibrating frequency of the cantilever.

As far as the register media, a CCD or a still camera, it is not able to follow the mechanical frequency we need to make a weighted average of each location, $W F(z)$. Actually, the cantilever will not spend the same time at each profile form, so it is necessary to use a weight function. After this, the first term of Eq. (3) becomes: 


$$
I_{1}(\theta)=\int_{A_{\min }}^{A_{\max }} W F(z)\left|\int_{0}^{H} e^{-i k\left(\theta+\beta(y, z)\left(y+\frac{z}{2}\right)+\delta(y, z)\right)} d y\right|^{2} d z
$$

and also Eq. (3) transforms into

$$
I=I_{1}(\theta)\left[\frac{\sin \left(\frac{1}{2} t k \sin \alpha\right)}{\frac{1}{2} t k \sin \alpha}\right]^{2}\left[\frac{\sin \left(\frac{1}{2} N b k \sin \alpha\right)}{\sin \left(\frac{1}{2} b k \sin \alpha\right)}\right]^{2} \text {. }
$$

\section{Experimental results vs simulated results}

As we expected from the diffraction model, the irradiance patterns obtained in the experimental set-up are distorted from the typical diffraction pattern given by a stationary array of slits. Actually, the experimental diffraction patterns obtained when the cantilevers are moving, show a widening with respect to those obtained for the stationary case. The movement is driven by the voltage generated by the piezoelectric transducer, so the cantilever movement is proportional to this voltage. Figure 5 shows the experimental images obtained from the cantilever array for the stationary case (upper image) and for the moving array (bottom figure) at $2 \mathrm{~V}$ of voltage. This voltage can be as high as $10 \mathrm{~V}$ for the cantilevers considered in this study. If we pay attention to the horizontal fringes of the central portion of the figures, we observe that the intensity is lower at the central portion of the diffractive pattern meanwhile the outer regions are enhanced. These effects are produced by the moving cantilever profile, and also appear in the simulated images. Finally, we may say that the experimental images are not symmetric. This asymmetry is generated the edge effects at the fixed portion of the cantilever array.

The simulated images are shown in Figs. 6 and 7 where two voltage values are used to simulate the irradiance distribution. In Fig. 7 we have saturated the maximum of the irradiance to shows better the diffraction pattern at the tails of the distribution. Using this procedure we may compare better the simulation with the experimental results, because they actually may contain saturated portions at the center of the distribution.
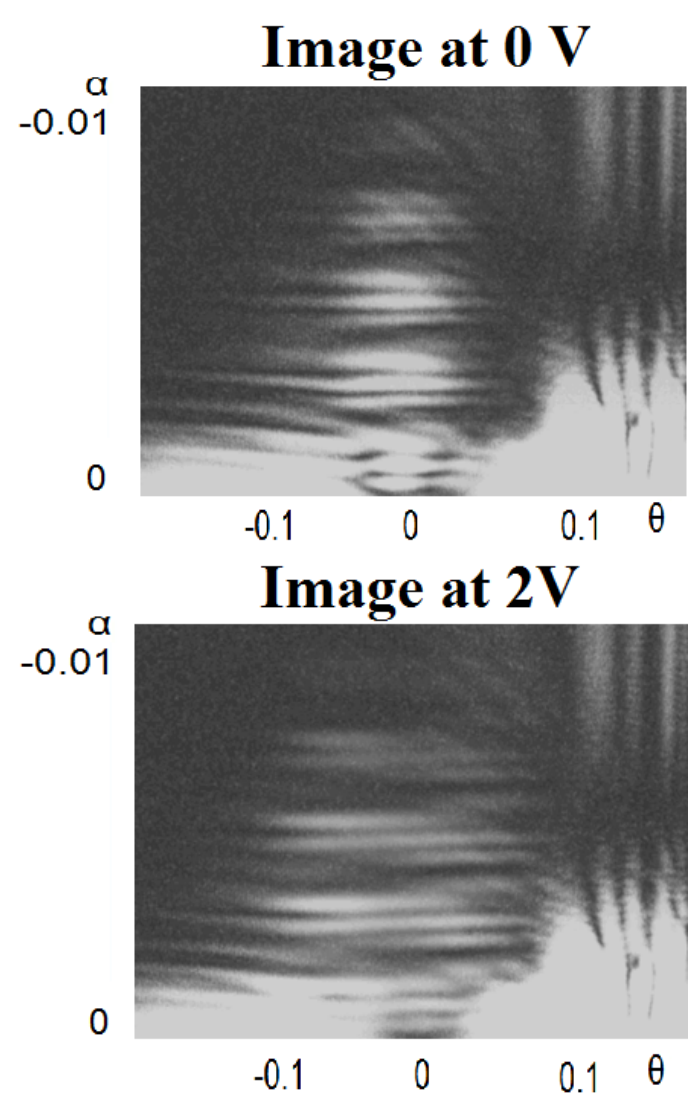

Fig. 5. Experimental images recorded at $0 \mathrm{~V}$ and $2 \mathrm{~V}$. The widening in the diffraction pattern is clearly revealed.
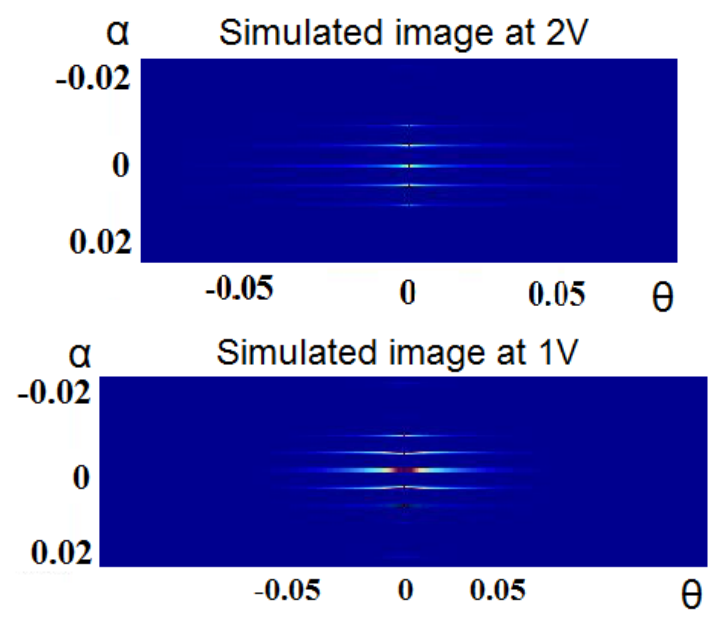

Fig. 6. Simulated images for two values of driving voltage. The diffractive pattern is wider as the voltage increases $(\alpha$ and $\theta$ are given in radians). 

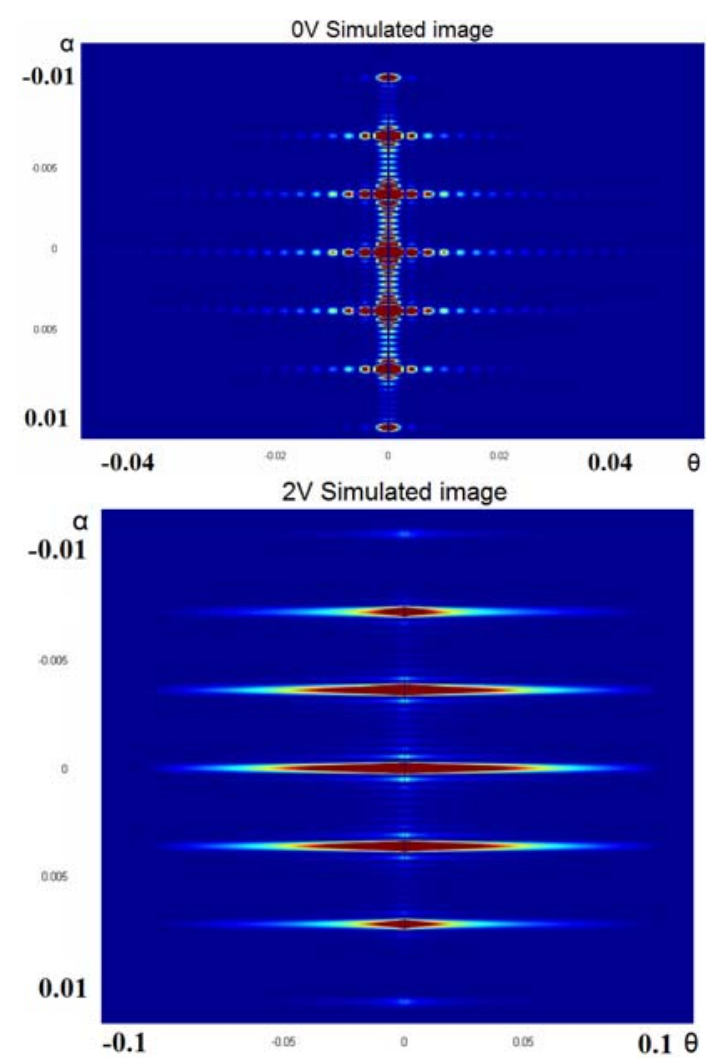

Fig. 7. Simulated images for two values of driving voltage. The diffractive patterns have been saturated for a better observation of the widening of the pattern $(\alpha$ and $\theta$ are given in radians).

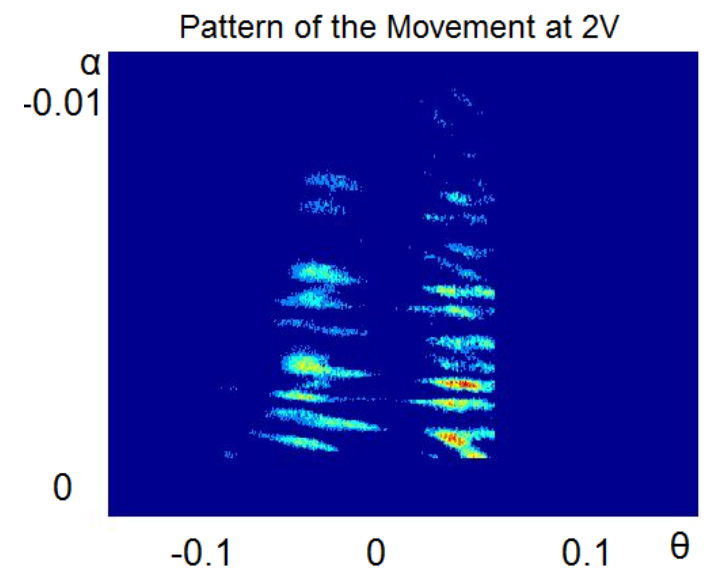

Fig. 8. Location of the portion of the image that changes when the applied a little voltage increment with respect to the $\mathrm{V}=0 \mathrm{~V}$ case. As the voltage is larger the changes are more noticeable. The image has been treated to remove noise.

\subsection{Comparative analysis of the diffraction pattern}

As we have seen before, when the cantilevers are vibrating at some given voltage, they will show a characteristic diffraction pattern. Then, by comparing the images obtained at different
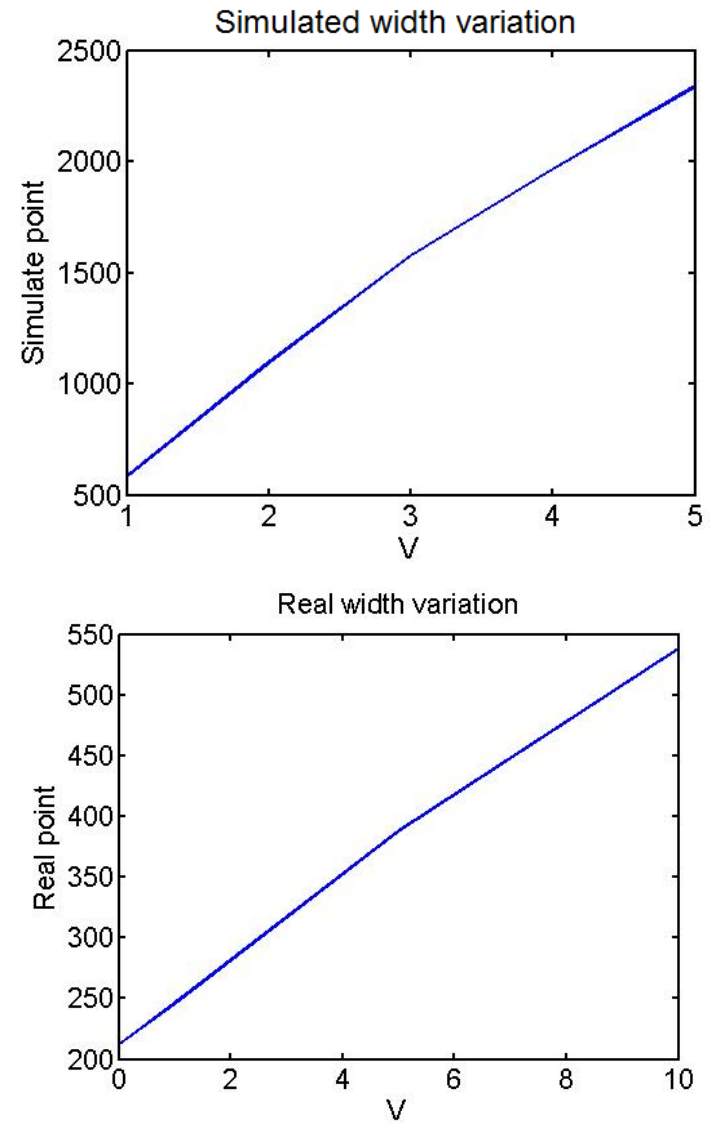

Fig. 9. Simulated (a) and experimental (b) variation of the pixel value as a function of the voltage.

voltages we may correlate these changes with the applied voltage as it is shown in Fig. 8. In this case we have removed the stationary pattern from the one obtained when the cantilevers are moving. Then, we may identify those portions of the diffractive pattern where the effect of the movement is analyzed. These portions are good candidates to locate a fast detector to follow the actual movement of the cantilever.

The image analysis provided in this contribution gives a clear perception of the changes produces by the movement, acting as a motion fingerprint. This method is good qualitatively but we may check its quantitative validity comparing the simulated and experimental images. The result of this comparison can be shown in Figs. 9(a) and 9(b). With the experimental and simulated results calculated in this case we may fit the signal a given point of the diffraction pattern as an almost-linear function of the applied voltage, with a slight decrease of the slope for high 
excitations. The points were this fitting apply are those located outside the stationary portion of the diffractive pattern. We mean as the stationary portion as the region where the diffractive patter appears when the cantilever is at rest. The deviation from the linear behavior at larger voltages can be produced by the dependence of the maximum angle in the movement of the cantilevers at high voltage levels excitation that is deviating from a linear dependence.

\section{Conclusion}

In this paper we have analyzed the results of a diffractive method to measure and characterize the movement of an array of cantilevers. The model uses the evaluation of the optical path for a vibrating cantilever. The movement of the cantilever is included and properly analyzed. The results from the model are compared with the experimental ones producing a good accordance.

\section{Acknowledgments}

This work has been done under the project ENE2009-14340-C02 of the Spanish Ministry of Science and Innovation. 